\title{
Highly Porous Piezoelectric PVDF Membrane as Effective Lithium Ion Transfer Channels for Enhanced Self-Charging Power Cell
}

\author{
Young-Soo Kim ${ }^{a, b}$, Yannan Xie ${ }^{a}$, Xiaonan Wen ${ }^{a}$, Sihong Wang ${ }^{a}$, Sang Jae Kim ${ }^{a, c}$, Hyun-Kon \\ Song ${ }^{b, *}$, and Zhong Lin Wang ${ }^{a, d, e, *}$
}

${ }^{a}$ School of Material Science and Engineering, Georgia Institute of Technology, Atlanta, GA 30332-0245, United States

${ }^{b}$ Ulsan National Institute of Science and Technology (UNIST), School of Energy and Chemical Engineering, 100 Banyeon-ri, Eonyang-eup, Ulsan 689-798, Republic of Korea

${ }^{c}$ Nanomaterials and system Lab., Department of Mechatronics Engineering, Jeju National University, Jeju 690-756, Republic of Korea.

${ }^{\mathrm{d}}$ Beijing Institute of Nanoenergy and Nanosystems, Chinese Academy of Science, Beijing, China

${ }^{\text {e }}$ Satellite Lab., MANA, WPI, National Institute For Materials Science, Japan.

\section{"Corresponding author.}

Tel.:+1 404894 8008. E-mail address: zlwang@gatech.edu (Z.L.Wang)

Tel.: +82 52217 2512. E-mail address: philiphobi@hotmail.com (H.-K.Song)

\section{Keywords}

Self-charging; Piezoelectric membrane; Energy harvesting; Energy storage; Lithium-ion secondary batteries

\begin{abstract}
A self-charging power cell (SCPC) is a structure that hybridizes the mechanisms for energy conversion and storage into one process through which mechanical energy can be directly converted into electrochemical energy. A key structure of an SCPC is the use of a polyvinylidene fluoride (PVDF) piezo-separator. Herein, we have fabricated a piezoelectric $\beta$-form PVDF
\end{abstract}


separator with a highly porous architecture by introducing $\mathrm{ZnO}$ particles. The electrochemical charge/discharge performance of this SCPC was greatly enhanced at lower discharge rates compared to highly stretched (high- $\beta$-content) or less porous PVDF membranes. The lower charge-transfer resistance of this well-developed porous piezo-separator is the main factor that facilitated the transport of $\mathrm{Li}^{+}$ions without sacrificing piezoelectric performance. This study reveals a novel approach for improving the performance of SCPCs.

\section{Introduction}

Piezoelectric nanogenerators have been recently garnering substantial attention in a variety of energy conversion and storage applications because of their remarkable intrinsic properties, such as a rapid electromechanical effect and relatively high force generation following a small external stimulus [1-6]. Among them, polyvinylidene fluoride (PVDF) with a predominantly $\beta$ phase crystal structure is investigated as the most widely available piezoelectric polymer for practical energy-generating devices [7-9]. Research utilizing the piezoelectricity of $\beta$-phase PVDF has been recently expanding into sustainable and renewable energy sources that can simultaneously exhibit energy harvesting and storage abilities, which has been accomplished by integrating the polarized PVDF polymer into an electrochemical system such as lithium-ion secondary batteries and supercapacitors [2,10-14]. The hybridization of the two separate energy processes (i.e., mechanically harvesting energy created from a piezoelectric potential and storing electrochemical energy induced by ion migration) provides an innovative method of selfcharging that can supply continuous power from the environment without the need for an external power source.

Rechargeable Li-ion batteries (LIBs), long positioned as an attractive power source in terms 
of energy storage, have aimed at maintaining their integrity over many charge/discharge cycles [15]. Thus, the introduction of a stabilized PVDF piezo-separator with the self-charging function as a replacement for the conventional polyethylene (PE) separator can be regarded as one of the most critical concerns required to maintain cell sustainability in LIBs. However, an important limitation exists concerning the practical application of a piezo-separator; the less- or non-porous structure of a PVDF piezo-separator causes ineffective migration of $\mathrm{Li}^{+}$ions from lithium transition metal oxide (e.g., $\mathrm{LiCoO}_{2}$ ) to a counter electrode such as graphite, despite its selfcharging capability through a mechanical-to-electrochemical process. Generally, to maximize piezoelectric performance as the means for self-charging, the PVDF film must have a high $\beta$ phase content, which can be achieved through uni- or biaxial stretching processes [16,17]. Unfortunately, these processes eliminate porosity within a PVDF membrane (Supporting information, S-1), which makes it difficult for the cells to charge and discharge galvanostatically, even at extremely low rates (Supporting information, S-2).

Thus, effective Li-ion transfer and outstanding piezoelectric output have become important considerations in the development of a $\beta$-phase PVDF piezo-separator with the self-charging feature. In this work, we focused on the design of a piezoelectric separator having well-defined porosity without sacrificing electrochemical performance. As the first step, we introduced $\mathrm{ZnO}$ particles to act as nucleating agents to induce the formation of the piezoelectric $\beta$-form and impart good solubility in acidic solution [8]. Second, the amount of $\mathrm{ZnO}$ particles was optimized because they strongly affect the porosity and flexibility of the PVDF piezo-separator. A selfcharging power cell (SCPC) fabricated with the optimized highly porous PVDF piezo-separator exhibited a charge/discharge profile (ca. $80 \%$ discharge capacity at $0.05 \mathrm{C}$ ) that was superior to that of a solution-cast PVDF separator without $\mathrm{ZnO}$ particles (ca. $10 \%$ at $0.05 \mathrm{C}$ ) or of a highly stretched commercial PVDF separator $(0 \%$ at $0.05 \mathrm{C})$. This is an important advance in PVDF 
piezo-separator development toward improved electrochemical performance and self-charging. It was evaluated in a conventional battery model; the schematic diagram in Figure 1a shows the structure of the SCPC wetted with a carbonate-based electrolyte containing $1 \mathrm{M} \mathrm{LiPF}_{6}$ salt. It used $\mathrm{LiCoO}_{2}$ as the cathode (Figure 1b) and artificial graphite as the anode (Figure 1c) with a porous $\mathrm{ZnO}$-free PVDF separator.

\section{Experimental section}

\section{Fabrication of a highly porous PVDF separator}

PVDF powder (Sigma-Aldrich, $\mathrm{M}_{\mathrm{w}}$ ca. 534,000) was fully dissolved in $N, N$-dimethylformamide (DMF; Sigma-Aldrich, $>99.9 \%)$ solvent $(10 \mathrm{wt} \%)$ at $23^{\circ} \mathrm{C}$. Then, $\mathrm{ZnO}$ particles (Alfa Aesar, 99.99\%, avg. 200nm size) with the same mass as the PVDF were added to the viscous PVDF solution. The PVDF/ZnO suspension was stirred at high speed and then treated in an ultrasonic bath for $1 \mathrm{~h}$ to provide a uniform dispersion. The mixture was then coated onto a glass substrate that had been previously cleaned with acetone; a razor blade provided a film of constant thickness. The coated mixture was dried at $60^{\circ} \mathrm{C}$ in an oven to evaporate the solvent. Then the prepared thin film was carefully immersed in aqueous concentrated hydrochloric acid (37 wt\%), etched for more than $12 \mathrm{~h}$ to completely eliminate $\mathrm{ZnO}$ particles, and washed several times with deionized water. Highly porous PVDF thin separators (30-40 $\mu \mathrm{m}$ thick) with well-constructed $\mathrm{Li}^{+}$paths were thus obtained. These separators were purged under an argon gas atmosphere for 1 $\mathrm{h}$ at room temperature before cell fabrication.

\section{Poling process to develop piezoelectric behavior}

To electrically polarize the porous PVDF separator, aluminum (Al) metal was deposited on both sides of the separator using an E-beam evaporator; the thickness of the Al coating was ca. 500 
$\mu \mathrm{m}$. The treated separator was then poled at room temperature for $2 \mathrm{~h}$ in an electric field of $20 \mathrm{~V}$ $\mu \mathrm{m}^{-1}$ to align the randomly organized $\beta$-form dipoles. No breakdown or fluctuation of the applied voltage occurred, indicating the stability of PVDF nanogenerators for poling process without any defect. The PVDF piezo-separator deposited with Al was sealed in the absence of electrolyte into a stainless steel 2016 size coin cell and the piezoelectric behavior of the poled PVDF separator was measured in a Faraday cage. The electrical potential output detected from the poled PVDF film was recorded with a Keithley 6514 Electrometer.

\section{Structural characterization of the PVDF thin film}

The surface morphology of the porous PVDF film was characterized using a Hitachi SU8010 scanning electron microscope (SEM). The crystalline phases present in the thin film were identified by Fourier transform infrared spectroscopy-attenuated total reflectance (FTIR-ATR) using an instrument equipped with a zinc selenide ( $\mathrm{ZnSe}$ ) crystal plate (Nicolet iS5 Spectrometer, Thermo Scientific) and by X-ray diffraction (XRD; X'Pert PRO, PANalytical B.V.) operating in continuous scanning mode with $10^{\circ}<2 \theta<60^{\circ}$.

\section{Electrochemical analysis and cell design}

Stainless steel 2016 coin full cells were employed with the poled PVDF porous separator exhibiting good wettability to the carbonate-based electrolyte. Cathodes were prepared by coating a slurry of $\mathrm{LiCoO}_{2}(\mathrm{KD} 10$, Umicore N.V.), 2 wt\% Super P (Timcal Graphite \& Carbon), and $2 \mathrm{wt} \%$ PVDF binder (KF7208, Kureha Corp.) on Al foil (15 $\mu \mathrm{m}$, Sam-A Aluminum Co., Ltd.). Anodes were prepared by coating a mixture of artificial graphite (Sumitomo-AR, Sumitomo Corp.) with $2 \mathrm{wt} \%$ Super P and $5 \mathrm{wt} \%$ PVDF binder (KF9130, Kureha Corp.) on copper $(\mathrm{Cu})$ foil $\left(10 \mu \mathrm{m}\right.$, Iljin Materials). All electrodes were dried at $90^{\circ} \mathrm{C}$ under vacuum after 
razor-blade coating. An electrolyte solution comprising $1 \mathrm{M} \mathrm{LiPF}_{6}$ (water content ca. 10 ppm;

Soulbrain MI) dissolved in ethylene carbonate (EC) and ethyl-methyl carbonate (EMC) (1:2 v:v) was dried with activated molecular sieves and filled into the cell before use. Cells were galvanostatically charged to $4.2 \mathrm{~V}$ and then discharged to $3.0 \mathrm{~V}$ at room temperature at a rate of $0.05 \mathrm{C}$ for stabilization. A battery analyzer (BST8-WA, MTI Corp.) was used for observing electrochemical performance and monitoring self-charging effect during mechanical compressive deformation.

\section{Electrochemical impedance measurement}

The impedance spectra of cells in the discharged state were obtained by applying $10 \mathrm{mV}$ ac amplitude in the frequency range of $500 \mathrm{kHz}$ to $10 \mathrm{mHz}$. For comparison at the same voltage, cells were initially charged at $4.2 \mathrm{~V}$ and discharged at $3 \mathrm{~V}$ galvanostatically after sufficient time for aging/wetting, i.e., more than $12 \mathrm{~h}$ after cell fabrication. All measurements were carried out at room temperature using a potentiostat (VersaSTAT3, Princeton Applied Research) equipped with an electrochemical impedance spectroscopy (EIS) analyzer.

\section{Results and discussion}

\section{Highly porous structure of $\beta$-form PVDF}

The motivation for this work resulted from the inadequate electrochemical performance during which highly stretched non- or weakly porous PVDF films displayed relatively serious charge/discharge capacities due to the absence of channels that would effectively allow the flow of $\mathrm{Li}^{+}$ions within the PVDF film (Supporting information S-2). Mechanically uniaxial stretching of PVDF film isothermally at high temperature (e.g., $80^{\circ} \mathrm{C}$ ) is a well-known technique to increase the $\beta$-phase content and thereby greatly improve the piezoelectric properties $[18,19]$. 
However, increasing the $\beta$-polymorph content in this manner decreases porosity and interferes in the smooth movement of $\mathrm{Li}^{+}$ions when the high contents of $\beta$-phase PVDF film is used as a separator in lithium-ion secondary batteries. Thus, we focused on how to develop a PVDF membrane having a well-defined porosity without sacrificing its electrochemical performance while maintaining a predominant $\beta$-phase form to exhibit piezoelectric characteristic. To simultaneously achieve these requirements, we uniformly dispersed $\mathrm{ZnO}$ particles in the viscous PVDF solution, which served several purposes: the $\mathrm{ZnO}$ particles acted as nucleating agents to provide the solution-cast PVDF film with a high $\beta$-phase content, and the uniform dispersion and facile removal of the particles by etching in acid solution did not damage the PVDF base film and provided well-defined $\mathrm{Li}^{+}$paths through the porous structure of the PVDF film. SEM images (Figure 1d) show many random holes well interconnected within the PVDF spherulitic crystal structure, indicating complete etching of the $\mathrm{ZnO}$ particles; XRD patterns (Figure 1e) show the growth of the $\beta$-phase PVDF and no peak signals corresponding to $\mathrm{ZnO}$ particles (Supporting Information S-3). The characteristic peaks at 840 and $1280 \mathrm{~cm}^{-1}$ observed in the FTIR-ATR spectrum also confirmed the formation of the $\beta$-phase in the ZnO-absent PVDF separator (Figure 1f).

\section{Basic mechanism for the SCPC}

Piezoelectric properties were observed for the highly porous Al-coated PVDF separator when it was sealed in a stainless steel 2016 coin cell in the absence of liquid electrolyte. The piezoelectric voltage output of the PVDF film was proportional to the externally applied force: the measured mean values were $2.31 \mathrm{~V}$ at $141 \mathrm{~mJ}, 3.84 \mathrm{~V}$ at $282 \mathrm{~mJ}$, and $6.21 \mathrm{~V}$ at $423 \mathrm{~mJ}$

(Figure 2a). The proportional response occurs because stress applied mechanically along a polarization axis increases strain in the porous PVDF film to generate a temporary flow of free 
electrons in the piezoelectric field, which results in a higher potential output. The average potentials were higher than those recently reported for other PVDF piezo-separators $[2,11,12,14]$. These enhanced values are attributable to a geometric strain effect induced by the highly porous structure of the PVDF that contributed to better alignment of dipoles and considerable displacement under external impact [8]. The piezoelectric potential profiles of this porous nanogenerator were also measured under periodic external compressive forces to confirm the piezoelectric polarities. The two potential outputs of the device shown in Figure 2b and 2c indicate that this porous PVDF separator demonstrated complete piezoelectric behavior with two opposite polarizations and maintained its stable polarization (i.e., behavior with no distinct degradation) under a constant compressive force (here, an external force of $353 \mathrm{~mJ}$ was applied). The bottom plot of each figure shows the features of the output signal generated during one mechanical compression cycle (i.e., a gradual decrease to the base voltage after apparent peak). The positive and negative output signals were respectively detected with the two opposite connections, indicating the authentic piezoelectric polarities in this porous PVDF piezoseparator.

A coin full cell comprising $\mathrm{LiCoO}_{2}$ as the cathode and artificial graphite as the anode, with a carbonate-based electrolyte, was fabricated to investigate the self-charging effect of the cells containing the porous PVDF piezo-separator (Supporting information, S-4). The typical selfcharging profile for this cell consisted of three processes (Figure 3a): (1) a periodic compression region that shows a self-charging profile with no external current input, (2) a galvanostatic discharge region, and (3) a subsequent equilibrium region at the open-circuit voltage $\left(\mathrm{V}_{\mathrm{oc}}\right)$. When a compression energy or force of $282 \mathrm{~mJ}$ [potential energy $(\mathrm{E})=m g h$; here, $m$ (mass of the object) was $288 \mathrm{~g}, g$ (acceleration due to gravity) was $9.8 \mathrm{~m} \mathrm{~s}^{-2}$, and $h$ (height) was $100 \mathrm{~mm}$ ] was applied to the coin cell at a frequency of $1 \mathrm{~Hz}$, the voltage of the cell increased from 1.2 to ca. $1.4 \mathrm{~V}$ over $200 \mathrm{~s}$. After completing the self-charging stimulus, the cell was galvanostatically 
discharged at a constant current of $0.01 \mathrm{~mA}(0.05 \mathrm{C})$ to $1.185 \mathrm{~V}$, when the discharge capacity required to return to the original potential $(1.2 \mathrm{~V})$ initiating self-charging was ca. $0.4 \mu \mathrm{Ah}$. Then, the cell voltage typically increased to the equilibrium voltage, $\mathrm{V}_{\text {oc }}$. The magnitude of the piezopotential and the self-charging of the device were directly proportional to the magnitude of the external impact. The two curves shown in Figure 3b illustrate the correlation between mechanical stress and piezo properties. When two mechanical forces having different potential energies of 141 and $282 \mathrm{~mJ}$ impacted the surface of the cell at a frequency of $1 \mathrm{~Hz}$, the device was self-charged to 1335 and $1400 \mathrm{mV}$, respectively, after 200 s. Galvanostatic discharge was carried out at a constant $0.01 \mathrm{~mA}$ current (here, the discharge capacity at a potential energy of $141 \mathrm{~mJ}$ after self-charging was ca. $0.18 \mu \mathrm{Ah}$ ), and a higher applied mechanical force enhanced the self-charging effect of the cell. When a constant force of $141 \mathrm{~mJ}$ was applied at a frequency of $1 \mathrm{~Hz}$ (Figure 3c), the increase in the potential generated from the iso-mechanical energy remained stable and the behavior was replicated over the two cycles shown. The cell that incorporated this porous PVDF piezo-separator was self-charged even under the stimulus of finger pressure, despite the hard stainless steel casing. Figure 3d shows that the self-charging effect enhanced by finger pressure almost reached the value obtained with an impact potential energy of $141 \mathrm{~mJ}$ (equivalent to dropping from a height of ca. $50 \mathrm{~mm}$ ). The highest mechanical force gave the greatest voltage enhancement of ca. $400 \mathrm{mV}$ and the discharge capacity of ca. 3.04 $\mu \mathrm{Ah}$, confirming the proportional relationship between self-charging and the magnitude of the externally applied mechanical force. Figure 3e shows the stability of the PVDF piezoseparator itself after mechanically shocking the device: no fluctuations occurred within the working potential range for ca. $3000 \mathrm{~s}$ at a constant charge and discharge rate of $0.01 \mathrm{~mA}$, indicating that the piezoelectric separator was not damaged or degraded by the mechanical stress.

The direction of the piezoelectric field generated by mechanical compression makes the 
PVDF piezo-separator maintain a positive piezopotential at the $\mathrm{LiCoO}_{2}$ surface and a negative piezopotential at the graphite surface because of close contact between the cathode and the anode. This piezoelectric field enables solvated $\mathrm{Li}^{+}$ions to easily migrate or transfer from the positive to the negative electrode through the porous PVDF piezo-separator, providing electron flow within the system. Accordingly, the working mechanism of the SCPC is an electrochemical process operated with the driving force induced from the piezoelectric potential of the $\beta$-phase PVDF separator. As illustrated in the schematic diagram of the Figure 4, three major reaction states associated with self-charging are working within the piezoelectric-driven electrochemical system: the initial state is an electrochemically stabilized region that is present before external compression is applied; in this state, the all electrode materials are wetted with liquid electrolyte and the PVDF piezo-separator only acts as an insulator to prevent electrical short between the cathode and the anode (Figure 4a). The second state is a self-charging region in which the piezoelectric field created by the PVDF piezo-separator forms along the $z$-axis from the cathode side of the system when compressive impact is applied to the cell surface. The piezoelectric potential of the porous PVDF separator causes $\mathrm{Li}^{+}$ions to migrate from the cathode to the anode through ion-conducting separator and electrolyte, resulting in the incorporation of $\mathrm{Li}^{+}$ions into the anode electrode. An electron takes the same pathway to maintain charge neutrality (Figure 4b); this migration represents complete conversion of mechanical energy into electrochemical energy. The final state is a force-releasing region in which small amounts of $\mathrm{Li}^{+}$ions diffuse backwards in the direction opposite their initial flow to reach balanced distribution when the external stimulus, which was the driving force for the unidirectional movement of $\mathrm{Li}^{+}$ions from cathode to anode, is removed (Figure 4c). Subsequently, the delithiated cathode and lithiated anode system that were self-charged from the piezoelectric field are electrochemically restabilized. Note that the piezopotential of the porous PVDF enables an effective redox reaction 
in the system, i.e., oxidation or delithiation $\left(\mathrm{LiCoO}_{2} \rightarrow \mathrm{Li}_{1-\mathrm{x}} \mathrm{CoO}_{2}+\mathrm{xLi}^{+}+\mathrm{xe}^{-}\right)$at the cathode and reduction or lithiation $\left(\mathrm{C}_{6}\right.$ (graphite) $\left.+\mathrm{xLi}^{+}+\mathrm{xe}^{-} \rightarrow \mathrm{Li}_{x} \mathrm{C}_{6}\right)$ at the anode. Another important consideration is that electrons may flow within the inner system without external circuit to neutralize the $\mathrm{Li}^{+}$cationic charge and maintain the net charge balance; this is a completely different mechanism from the typical charge/discharge system of LIBs in which the flow of electron is only available through an external circuit. In the general concept regarding energystorage of Li-ion chemistries, the travel of electrons through the inner cell system can be mainly related to a safety issue due to the possibility of an internal short-circuit resulting from the thermally abusive condition [20-22]. However, in the self-charging cell, electron flow through the PVDF piezo-separator is at the micro- or milli-ampere level and never leads to thermal side reaction during mechanical stimulus. Furthermore, the high melting point $\left(\mathrm{T}_{\mathrm{m}}\right)$ of PVDF reduces the risk of cell degradation. The existence of an electron leakage mentioned in several literatures also supports the possibility of a reversible electron transfer through inner system [23,24]. The recovery profile shown in Figure 3e demonstrates the stability of the SCPC toward electron transfer through the inner electrochemical system.

\section{Electrochemical properties of the porous PVDF piezo-separator}

As noted above, electrochemical performance is crucial in considering self-charging nanogenerators for use in practical lithium-ion secondary battery applications. A PVDF piezoseparator, even with the self-charging capability described in the previous section, may have only limited applications if cell performance were diminished in the presence of the piezoseparator. Porosity is the most important characteristic that controls the effective migration of $\mathrm{Li}^{+}$ ions within the electrochemical system. Most conventional or solution-cast PVDF films (Supporting information S-1, S-2) have non- or less-porous architectures, which prevent the 
movement of $\mathrm{Li}^{+}$ions even if the film has a high $\beta$-polymorph content or a piezoelectric contribution that leads to self-charging. Thus, providing a porous structure to the PVDF separator without a serious loss of $\beta$-content with its aligned dipole configuration is beneficial in terms of the simultaneous satisfaction of both self-charging and electrochemical performance. The normalized capacity (Q) of a coin full cell consisting of two electrodes $\left(\mathrm{LiCoO}_{2}\right.$ and graphite), a PVDF separator, and a liquid carbonate-based electrolyte (Figure 5a) illustrates the significant difference in initial performance when a weakly porous and highly porous PVDF piezo-separator was used, respectively. The enhancements in $\mathrm{Q}$ at the current rate of $0.05 \mathrm{C}$ for charging $\left(\mathrm{Q}_{\mathrm{c}}\right)$ and discharging $\left(\mathrm{Q}_{\mathrm{d}}\right)$ (i.e., 84.6 and $73.6 \%$, respectively) were much larger than that of a typical solution-cast PVDF film made without the $\mathrm{ZnO}$ particle treatment. The PVDF piezo-separator whose porosity was well-defined by the $\mathrm{ZnO}$ particles $\left(\mathrm{PVDF}_{\mathrm{w} . \mathrm{p}}\right)$ had an outstanding $\mathrm{Q}_{\mathrm{d}}$ of ca. $82 \%$, while the less porous separator $\left(\mathrm{PVDF}_{\text {wo.p }}\right)$ had a $\mathrm{Q}_{\mathrm{d}}$ of only ca. $8 \%$ because of high ohmic resistance (or I-R drop) and serious overpotential. Except for the initial irreversible capacity accompanying large Li loss over three cycles at $0.05 \mathrm{C}$, the $\mathrm{PVDF}_{\mathrm{w} . \mathrm{p}}$ coulombic efficiency gradually increased, averaging $>98 \%$, during cycling regardless of the current rate (Figure 5b). The normalized discharge profile $\left(\mathrm{Q}_{\mathrm{d}}^{\prime}\right)$ based on the initial discharge capacity (the bottom plot of Figure 5b) exhibited there can be a possibility of capacity fading due to the large I-R drop and concentration polarization normally detected at high rates: $\mathrm{Q}^{\prime}{ }_{\mathrm{d}}$ was ca. $85 \%$ at $0.3 \mathrm{C}$ compared with ca. $95 \%$ at $0.05 \mathrm{C}$. This is still a great improvement over conventional solution-cast $\mathrm{PVDF}_{\text {wo.p }}$, which suffers from a difficult charge/discharge process. Such porosity-induced behavior, which is related to the resistance to inhibit ionic conductivity of $\mathrm{Li}^{+}$ions, is also supported by the results of EIS over the frequency range of $500 \mathrm{kHz}$ to $10 \mathrm{mHz}$ (Figure 5c). The impedance of $\mathrm{PVDF}_{\text {w.p }}$ was significantly lower than that of $\mathrm{PVDF}_{\text {wo.p }}$; the charge-transfer resistance $\left(\mathrm{R}_{\mathrm{ct}}\right)$ of the cathode and anode in the cell with $\mathrm{PVDF}_{\mathrm{w} . \mathrm{p}}$ in the 
discharged state (3.1 V) was ca. $200 \Omega$, while $\mathrm{PVDF}_{\text {wo.p }}$ had an $\mathrm{R}_{\mathrm{ct}}$ of ca. $3000 \Omega$, which is more than 10 times higher than that of $\mathrm{PVDF}_{\mathrm{w} . \mathrm{p}}$. This unfavorable result for the $\mathrm{PVDF}_{\text {wo.p }}$ piezoseparator is consistent with the serious capacity fading detected in the initial charge/discharge profiles shown in Figure 5a; the less porous structure is the main reason for the large impedance because it interferes with the effective mobility of $\mathrm{Li}^{+}$ions within the cell. The calendar life of the porous piezo-separator at $23^{\circ} \mathrm{C}$ (Figure 5d) shows that it maintained a stable discharge capacity at ca. $92 \%$ and a coulombic efficiency $>98 \%$ during continuous charge/discharge processes at a rate of $0.1 \mathrm{C}$ when the initial large irreversible capacity was excluded.

\section{Conclusions}

We investigated a highly porous PVDF film as an effective piezo-separator for enhanced SCPCs. The well-defined porous structure stabilized the device, reduced charge transfer resistance, and prevented serious capacity decay, simultaneously enabling a self-charging effect induced by the piezopotential. Our strategy suggests a practical research direction for SCPCs utilizing the piezoelectric characteristics of predominantly $\beta$-phase PVDF, which enables direct conversion of mechanical energy into electrochemical energy without an external electrical source. In contrast to the conventional PVDF piezo-separator, which suffers from substantial capacity loss, the cells using the highly porous PVDF separator described herein were tuned to render them completely immune to electrochemical defects. Critical advantages exist over previous approaches to the application of PVDF piezo-separators in LIBs. This study, based on a porous architecture of $\beta$ phase PVDF, suggests a useful approach to overcome some serious limitations of current stateof-the-art SCPCs. 


\section{Acknowledgments}

This work was supported by the U.S. Department of Energy, Office of Basic Energy Sciences (DE-FG02-07ER46394), MANA, National Institute for Materials Science, Japan, the Thousands Talents, China program for pioneer researchers and his/her innovation team, and National Research Foundation of Korea (2013R1A1A2064471). Y.-S. Kim was supported by a fellowship program from UNIST

\section{Supporting information}

Thermal stretching of solution-cast PVDF film at $80^{\circ} \mathrm{C}$; surface morphology and spectroscopic analysis for the $\beta$-phase in the film; electrochemical performance at a rate of $0.05 \mathrm{C}$ for the $\mathrm{LiMn}_{2} \mathrm{O}_{4}$-based coin half-cells incorporating a commercial PVDF or a PE separator; X-ray diffraction analysis of $\mathrm{ZnO}$-present and -absent PVDF separators. 


\section{Vitae}

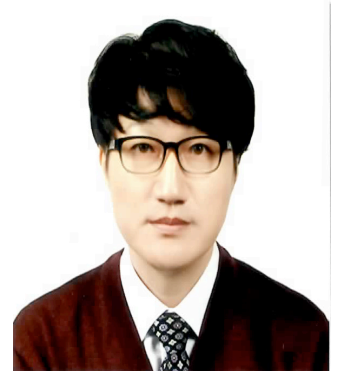

Dr. Young-Soo Kim received his Ph.D. degree in Energy Engineering at the UNIST in Korea in 2014; he is now a postdoctoral researcher in the Materials Science and Engineering at the Georgia Institute of Technology. He has worked in the field of lithium-ion secondary batteries since 2001, in particular focusing on the thermal-stable electrolytes, functional binders, and safety-reinforced separators. His current research interest is the self-charging power cells using piezoelectric separator.

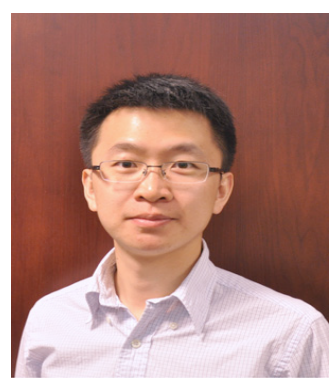

Yannan Xie is a Ph.D. candidate in the Department of Physics at Xiamen University and also a visiting student in Prof. Zhong Lin Wang's group in the School of Materials Science and Engineering at the Georgia Institute of Technology. His research interests include synthesis of nanomaterials, nanogenerators, self-powered systems, and optoelectronic devices.

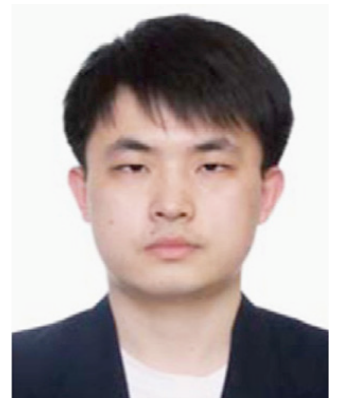

Xiaonan Wen received his B.S. degree in physics from Peking University, China, in 2010. He is currently a Ph.D. candidate in the School of Materials Science and Engineering at the Georgia Institute of Technology. His research interests include the synthesis of functional nanomaterials, energy harvesting using piezoelectric and triboelectric generators, self-powered nanosystems, piezo-electronics, and piezo-optoelectronics based on $\mathrm{ZnO}$ and $\mathrm{GaN}$ for novel transistors and devices and their integration into functional systems. 


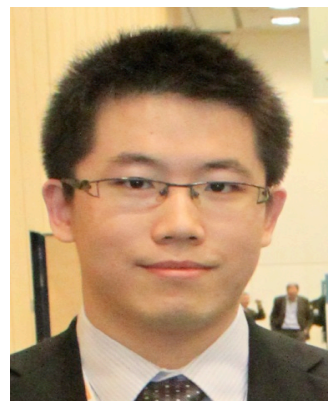

Dr. Sihong Wang is currently a postdoctoral fellow in Prof. Zhong Lin Wang's group at the Georgia Institute of Technology. He obtained his Ph.D. degree in Materials Science and Engineering from the Georgia Institute of Technology in 2014 under the supervision of Prof. Wang, and his B.S. in Materials Science and Engineering from Tsinghua University, China, in 2009. His research mainly focuses on nanomaterial-based mechanical energy harvesting and energy storage, self-powered systems, nanogenerator-based sensors, and piezotronics.

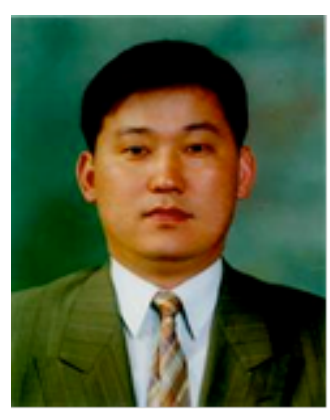

Prof. Sang Jae Kim is a professor in the Department of Mechatronics Engineering and the Department of Advanced Convergence Technology and Science at Jeju National University, Korea. He received his Ph.D. degree in Electrical Communication Engineering from Tohoku University, Japan. He was a visiting research scholar in materials science at the University of Cambridge and at the Georgia Institute of Technology, and a senior researcher at the National Institute of Materials Science (NIMS), Japan. His research disciplines are based on nanomaterials and systems for energy and electronics applications, covering Josephson devices, MEMS, and nanobiosensors.

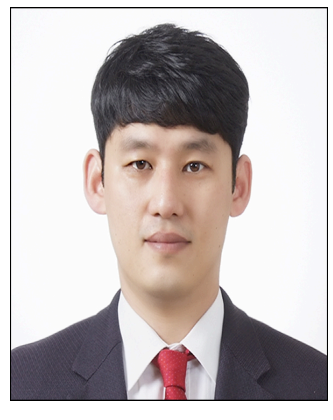

Prof. Hyun-Kon Song is an associate professor in the Interdisciplinary School of Green Energy at UNIST, Korea. He received his Ph.D. degree in Chemical Engineering from POSTECH, Korea. He was a postdoctoral researcher at Brown University and Seoul National University, and a senior researcher at LG Chem/Research Park. His research disciplines are based on 
electrochemistry and materials chemistry, covering the synthesis of electroactive materials and the novel design of electrochemistry-based devices.

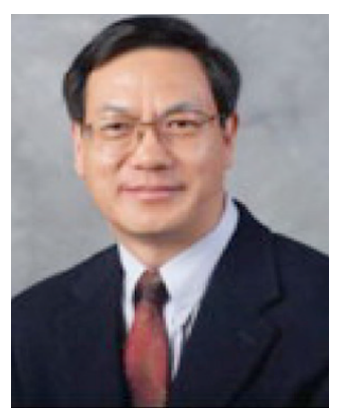

Zhong Lin (ZL) Wang received his Ph.D. degree from Arizona State University in physics. He holds the Hightower Chair in Materials Science and Engineering and is Regent's Professor, Engineering Distinguished Professor, and Director of the Center for Nanostructure Characterization at Georgia Tech. Dr. Wang has made original and innovative contributions to the synthesis, discovery, characterization, and understanding of fundamental physical properties of oxide nanobelts and nanowires, as well as applications of nanowires in energy sciences, electronics, optoelectronics, and biological science. His discovery and breakthroughs in developing nanogenerators established the principles and technological road map for harvesting mechanical energy from the environment and from biological systems for powering personal electronics. His research on self-powered nanosystems has inspired a worldwide effort in academia and industry for studying energy in micro/nanosystems, which is now a distinct discipline in energy research and future sensor networks. He coined and pioneered the field of piezotronics and piezophotonics by introducing the piezoelectric potential-gated charge transport process into the fabrication of new electronic and optoelectronic devices. Details can be found at http://www.nanoscience.gatech.edu. 
(a)
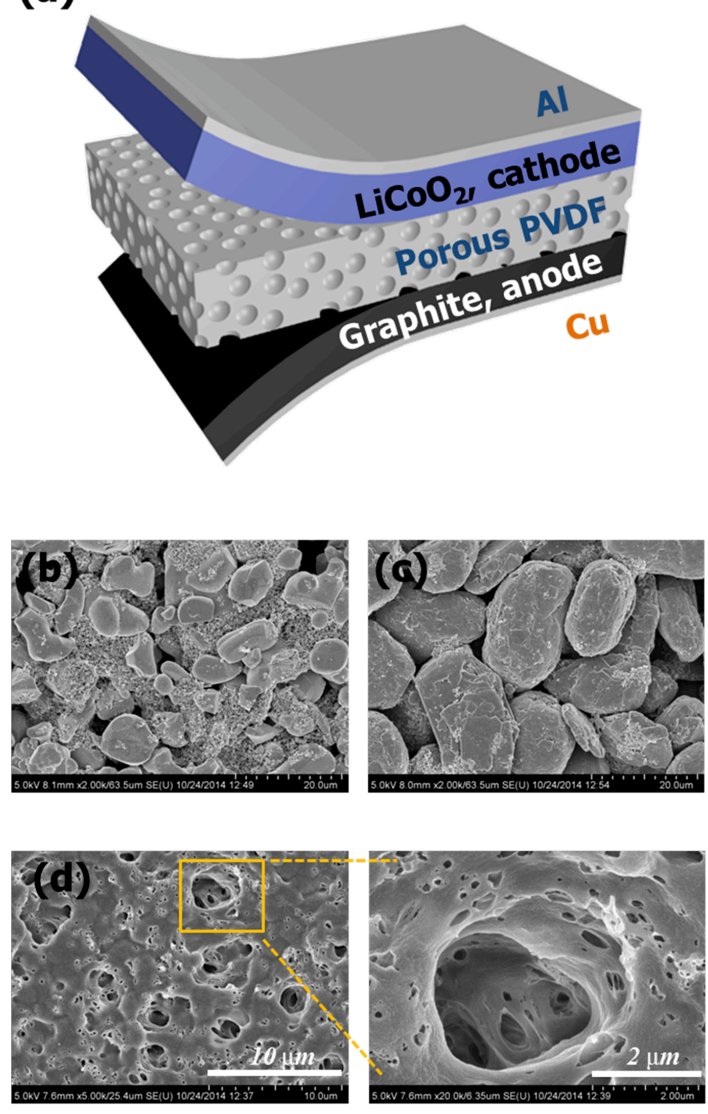
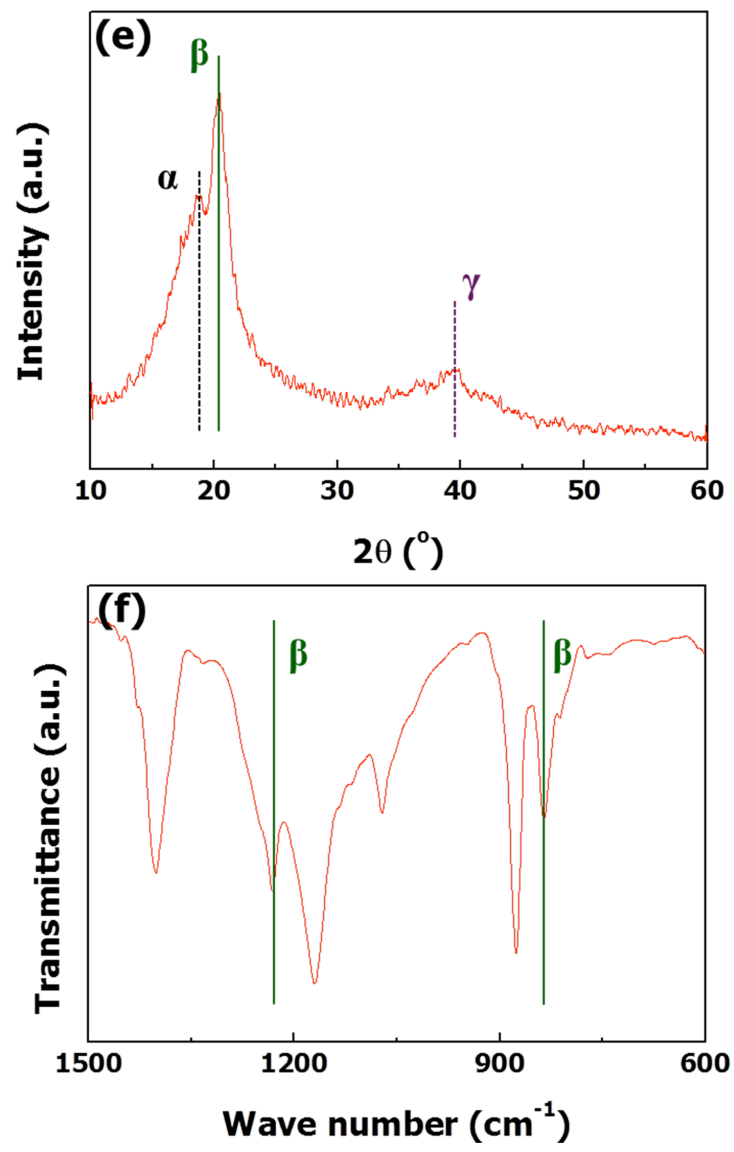

Figure 1 (a) Schematic diagram of an $\mathrm{SCPC}$ consisting of $\mathrm{LiCoO}_{2}$ as the cathode, artificial graphite as the anode, and a porous PVDF piezo-separator. Morphological image of the cathode (b) coated on an $\mathrm{Al}$ sheet and the anode (c) coated on a $\mathrm{Cu}$ sheet. (d) PVDF surface image showing the amorphous macro- and mesoporous structure at low (left) and high (right) magnifications. (e) XRD pattern showing complete elimination of the $\mathrm{ZnO}$ particles and growth of the $\beta$-phase PVDF, which is supported by the observed FTIR-ATR peaks (f). 

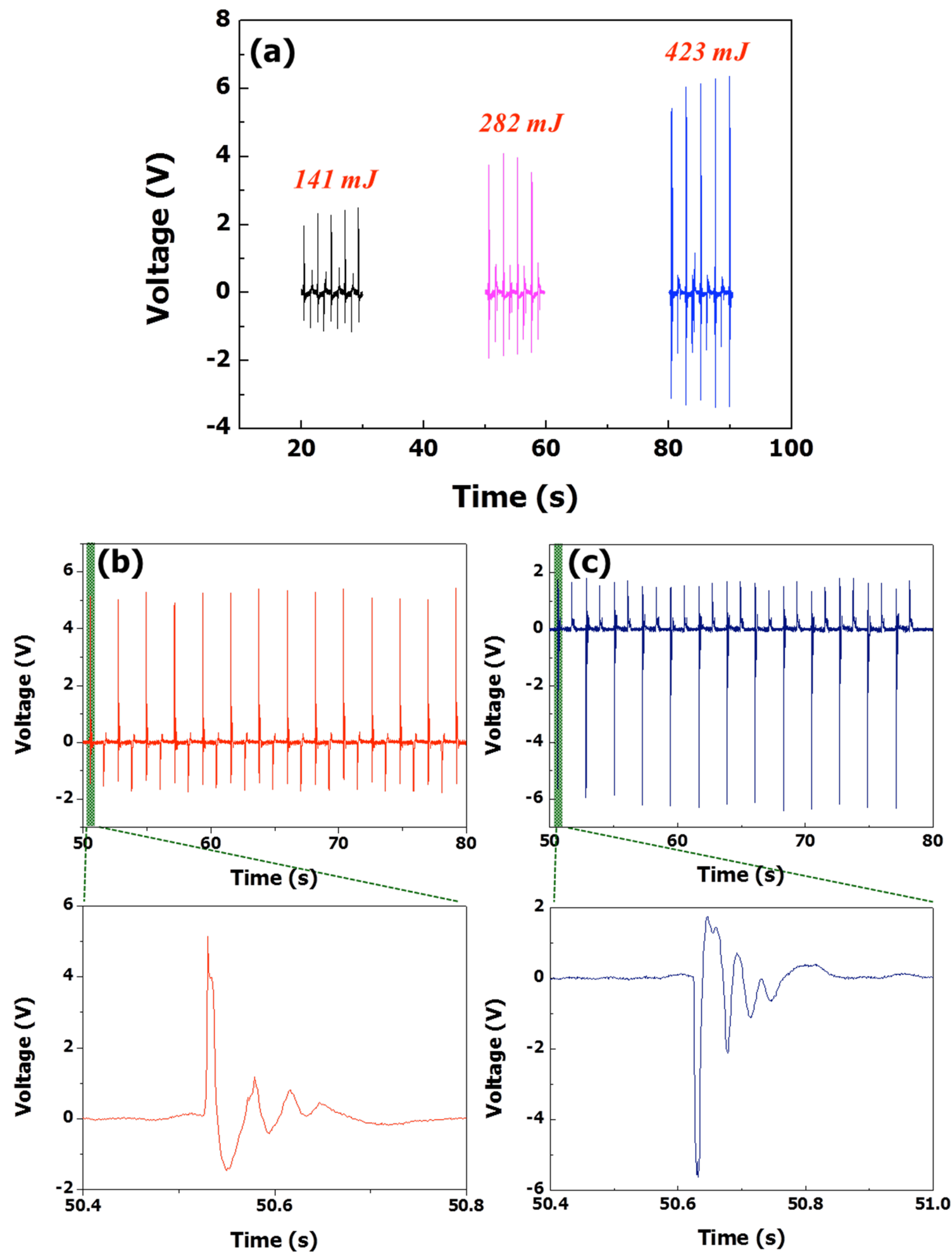

Figure 2 (a) Periodic piezoelectric voltage output of the porous PVDF piezo-separator under externally applied forces of 141,282 , and $423 \mathrm{~mJ}$ in the absence of electrolyte. The magnitude of 
the voltage peak is proportional to the applied mechanical force. (b and c) Characteristic piezoelectric signals obtained in the two opposite polarizations for an externally applied force of $353 \mathrm{~mJ}$. The lower graphs show the voltage output generated during one mechanical compression cycle and confirm the piezoelectric behavior of the porous PVDF separator. 

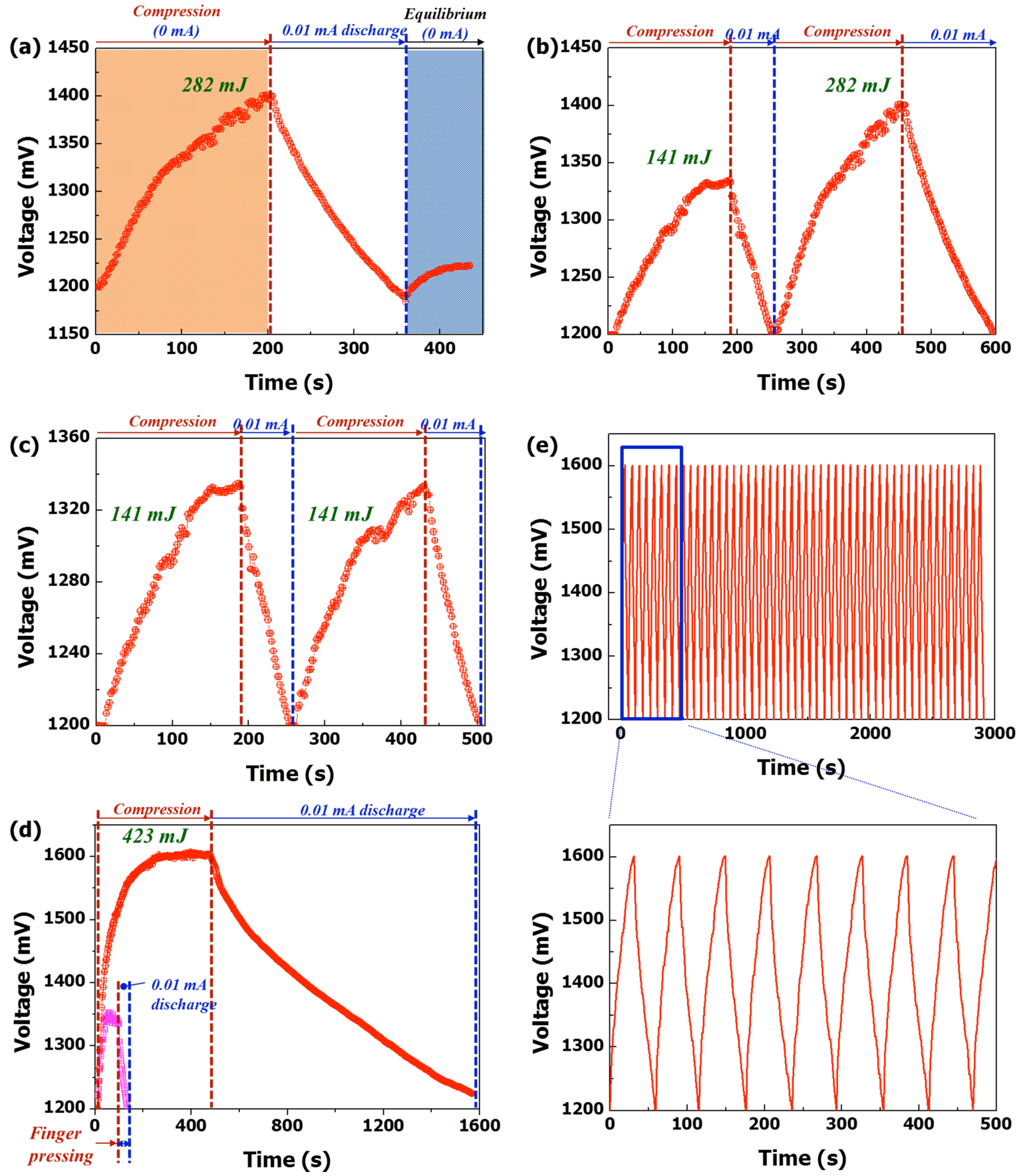

Figure 3 (a) A typical self-charging profile comprising three regions: a compression region inducing self-charging over an initial $200 \mathrm{~s}$ when continuous mechanical compression of $282 \mathrm{~mJ}$ was applied at $1 \mathrm{~Hz}$ to the cell, a galvanostatic $(0.01 \mathrm{~mA})$ discharge region immediately following the self-charging, and an equilibrium region with no current input. (b and c) Two self-charging cycles with different and identical applied forces, respectively. (d) Comparison of self-charging 
behavior obtained using two different compression methods: finger pressure, which is similar to an applied mechanical force of $141 \mathrm{~mJ}$, and a high mechanical force of $423 \mathrm{~mJ}$, which gave the greatest voltage increase of ca. $400 \mathrm{mV}$. (e) Galvanostatic charge/discharge profile within the self-charging range of 1.2-1.6 V. The expanded lower profile shows the behavior over $500 \mathrm{~s}$, which confirmed that no damage occurred to the porous PVDF piezo-separator.

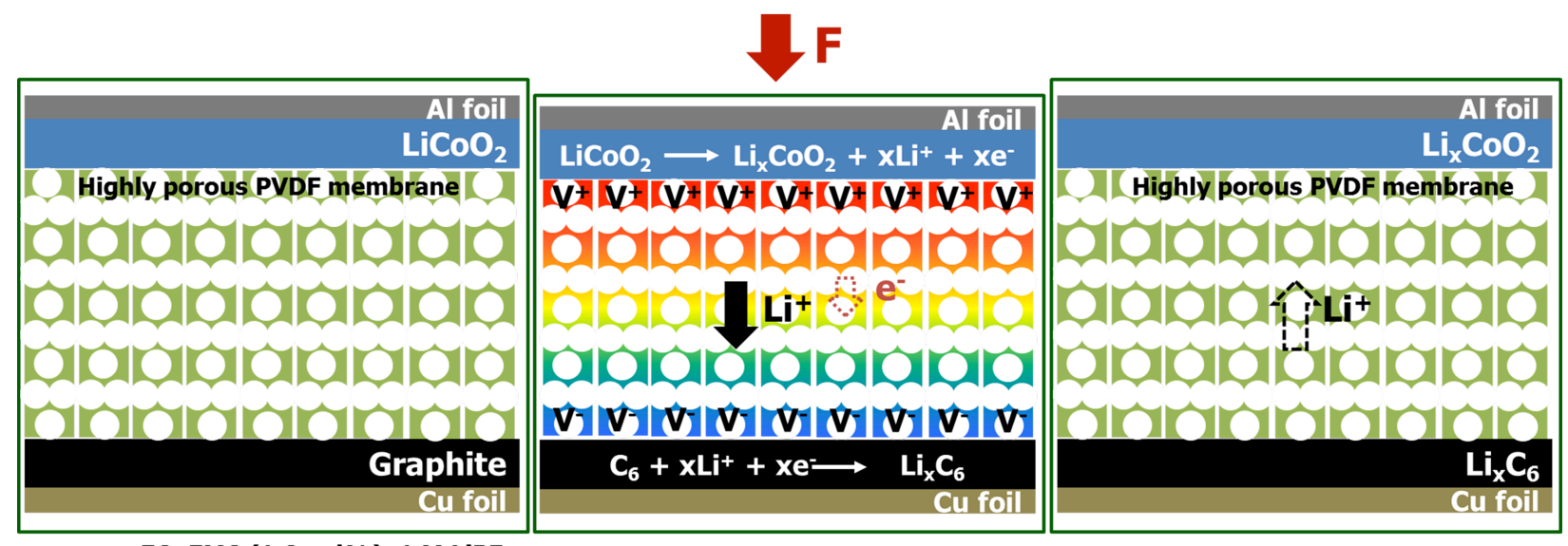

EC: EMC (1:2 vol\%), 1 M LiPF

(a) Initial state

(b) Applied force

(c) Released force

Figure 4 Schematic self-charging mechanism induced by a porous PVDF piezo-separator in Liion secondary batteries. (a) Initial state before the application of compressive strain in which the piezo-separator is wetted by the electrolyte. (b) With the applied external force, the PVDF piezoseparator generates positive and negative piezoelectric potentials at the cathode and the anode, respectively. The piezoelectric field generated up and down in the system drives the migration of $\mathrm{Li}^{+}$ions through the porous piezo-separator from the cathode to the anode with accompanying electron transfer. (c) After removing the applied force, the device reaches the equilibrium $V_{o c}$, which drives the diffusion of $\mathrm{Li}^{+}$ions backwards because of the disappearance of the piezoelectric field. 
(a)
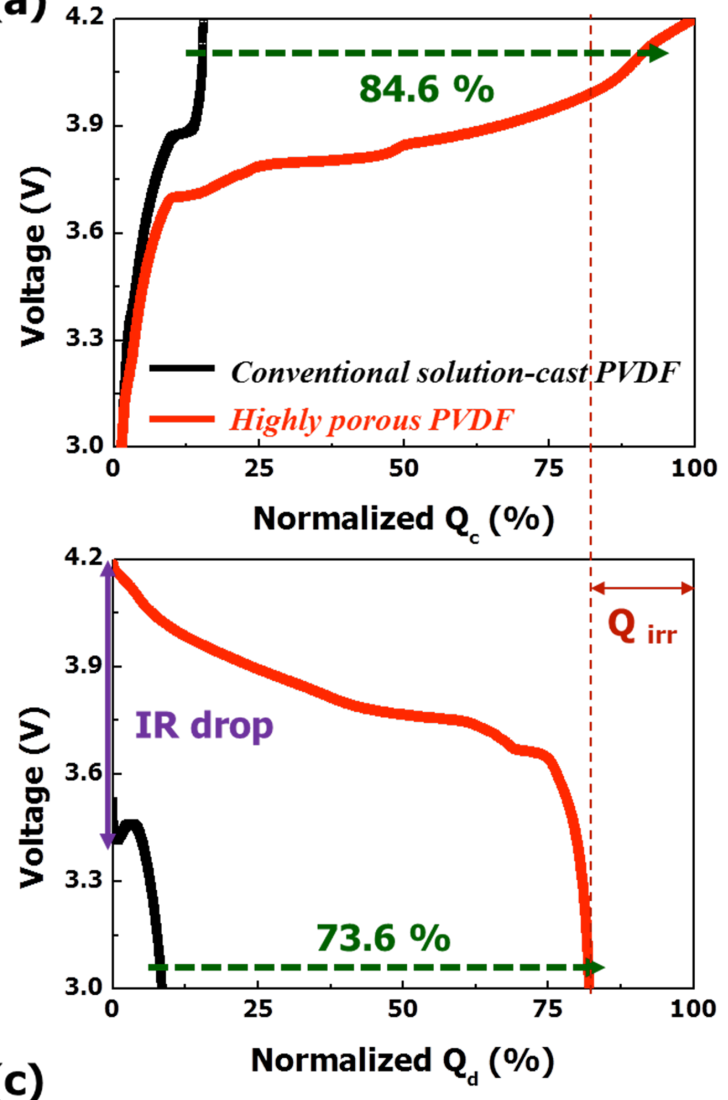

(c)

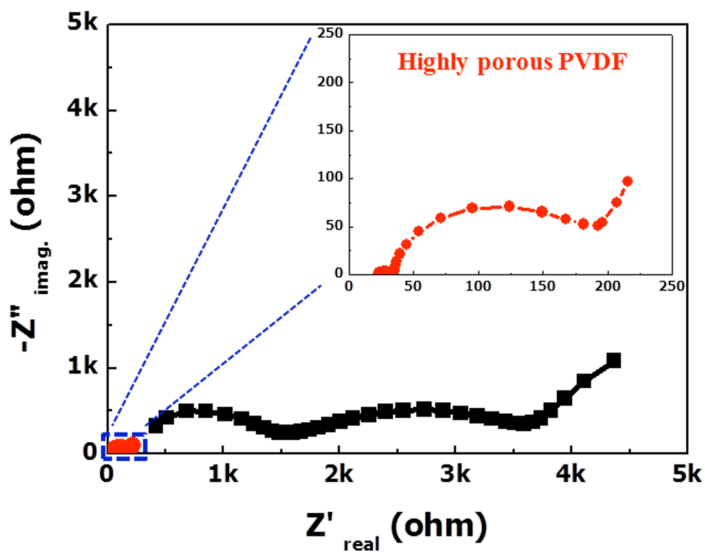

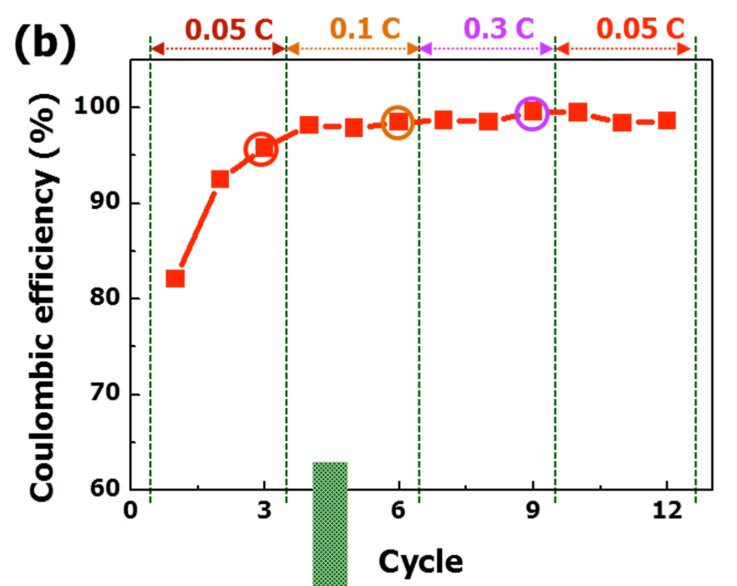

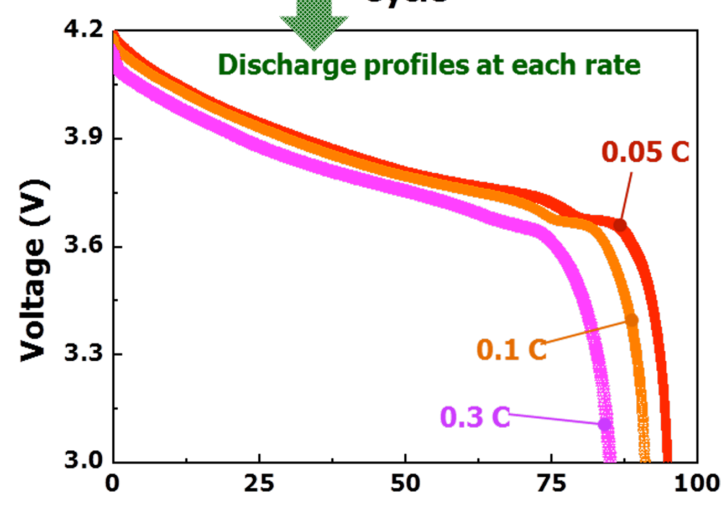

(d)

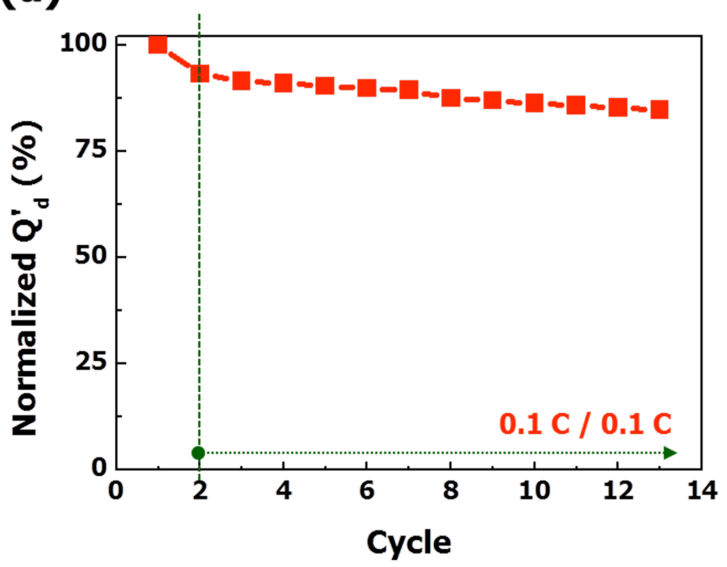

Figure 5 (a) Normalized initial charge $\left(Q_{c}\right.$, upper plot) and discharge capacity $\left(Q_{d}\right.$, lower plot $)$ at a current rate of $0.05 \mathrm{C}$ for a solution-cast low-porosity PVDF piezo-separator (black line, $\left.\mathrm{PVDF}_{\text {wo.p }}\right)$ and a highly porous PVDF piezo-separator (red line, $\left.\mathrm{PVDF}_{\mathrm{w} . \mathrm{p}}\right)$. (b) Coulombic efficiency of $\mathrm{PVDF}_{\mathrm{w} . \mathrm{p}}$ as a function of current rate; the sequence follows three steps per $\mathrm{C}$ rate (here, the charge/discharge current at each step is the same); the discharge profile at the third 
cycle of each C rate is detailed on the bottom plot. (c) Nyquist plots of the impedance measured at a discharged potential of ca. 3.1 V; $\mathrm{PVDF}_{\text {wo.p }}$ has serious solution and charge-transfer resistance $\left(\mathrm{R}_{\mathrm{ct}}\right)$ compared with the impedance of $\mathrm{PVDF}_{\mathrm{w} . \mathrm{p}}$ plotted in the inset. (d) Calendar life of the porous piezo-separator at $23^{\circ} \mathrm{C}$. The discharge capacity $\left(\mathrm{Q}_{\mathrm{d}}^{\prime}\right.$ normalized on the basis of the initial discharge capacity) was monitored in the constant current charge/discharge mode (0.1 C) after an initial low current $(0.05 \mathrm{C})$ was used in the first cycle to stabilize the electrode system.

\section{References}

[1] Y. Hu, L. Lin, Y. Zhang, Z.L. Wang, Advanced Materials 24 (2012) 110.

[2] X. Xue, S. Wang, W. Guo, Y. Zhang, Z.L. Wang, Nano letters 12 (2012) 5048.

[3] G. Zhu, A.C. Wang, Y. Liu, Y. Zhou, Z.L. Wang, Nano letters 12 (2012) 3086.

[4] M. Lee, C.Y. Chen, S. Wang, S.N. Cha, Y.J. Park, J.M. Kim, L.J. Chou, Z.L. Wang, Advanced Materials 24 (2012) 1759.

[5] R. Yang, Y. Qin, L. Dai, Z.L. Wang, Nature Nanotechnology 4 (2008) 34.

[6] Z.L. Wang, Advanced Functional Materials 18 (2008) 3553.

[7] C. Chang, V.H. Tran, J. Wang, Y.-K. Fuh, L. Lin, Nano letters 10 (2010) 726.

[8] Y. Mao, P. Zhao, G. McConohy, H. Yang, Y. Tong, X. Wang, Advanced Energy Materials 4 (2014) 1301624.

[9] C. Sun, J. Shi, D.J. Bayerl, X. Wang, Energy \& Environmental Science 4 (2011) 4508.

[10] J. Porhonen, S. Rajala, S. Lehtimaki, S. Tuukkanen, IEEE Transactions on Electron Devices 61 (2014) 9.

[11] L. Xing, Y. Nie, X. Xue, Y. Zhang, Nano Energy 10 (2014) 44.

[12] X. Xue, P. Deng, B. He, Y. Nie, L. Xing, Y. Zhang, Z.L. Wang, Advanced Energy Materials 4 (2014) 1301329.

[13] X. Xue, P. Deng, S. Yuan, Y. Nie, B. He, L. Xing, Y. Zhang, Energy \& Environmental Science 6 (2013) 2615.

[14] Y. Zhang, Y. Zhang, X. Xue, C. Cui, B. He, Y. Nie, P. Deng, Z.L. Wang, Nanotechnology 25 (2014) 105401.

[15] H. Lee, M. Yanilmaz, O. Toprakci, K. Fu, X. Zhang, Energy \& Environmental Science (2014).

[16] J. Gomes, J.S. Nunes, V. Sencadas, S. Lanceros-Mendez, Smart Materials and Structures 19 (2010) 065010.

[17] L. Li, M. Zhang, M. Rong, W. Ruan, RSC Advances 4 (2014) 3938. 
[18] V. Sencadas, R. Gregorio Jr, S. Lanceros-Méndez, Journal of Macromolecular Science® 48 (2009) 514.

[19] H.-J. Ye, L. Yang, W.-Z. Shao, S.-B. Sun, L. Zhen, RSC Advances 3 (2013) 23730.

[20] P. Balakrishnan, R. Ramesh, T. Prem Kumar, Journal of Power Sources 155 (2006) 401.

[21] S.-i. Tobishima, J.-i. Yamaki, Journal of Power Sources 81 (1999) 882.

[22] H. Maleki, G. Deng, A. Anani, J. Howard, Journal of The electrochemical society 146 (1999) 3224.

[23] R.P. Ramasamy, J.-W. Lee, B.N. Popov, Journal of power sources 166 (2007) 266.

[24] R. Yazami, Y.F. Reynier, Electrochimica acta 47 (2002) 1217.

The English in this document has been checked by at least two professional editors, both native speakers of English. For a certificate, please see:

http://www.textcheck.com/certificate/0efGxE 